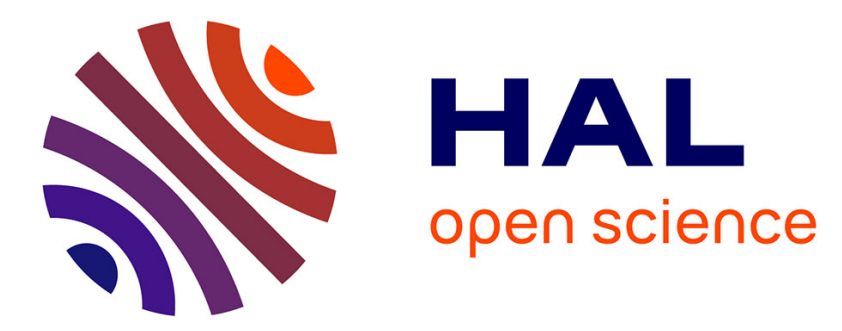

\title{
Mausolée et four crématoire gallo-romains à Mackwiller (Bas-Rhin) \\ Jean-Jacques Hatt
}

\section{To cite this version:}

Jean-Jacques Hatt. Mausolée et four crématoire gallo-romains à Mackwiller (Bas-Rhin). Gallia - Fouilles et monuments archéologiques en France métropolitaine, 1967, 25 (1), pp.75-85. 10.3406/galia.1967.2465. hal-01934404

\section{HAL Id: hal-01934404 \\ https://hal.science/hal-01934404}

Submitted on 3 Mar 2020

HAL is a multi-disciplinary open access archive for the deposit and dissemination of scientific research documents, whether they are published or not. The documents may come from teaching and research institutions in France or abroad, or from public or private research centers.
L'archive ouverte pluridisciplinaire HAL, est destinée au dépôt et à la diffusion de documents scientifiques de niveau recherche, publiés ou non, émanant des établissements d'enseignement et de recherche français ou étrangers, des laboratoires publics ou privés.

\section{(이) $\$$}

Distributed under a Creative Commons Attribution - NonCommercial - NoDerivatives $\mid 4.0$ 


\title{
MAUSOLÉE ET FOUR CRÉMATOIRE GALLO-ROMAINS A MACKWILLER (BAS-RHIN)
}

\author{
par Jean-Jacques HATT
}

\begin{abstract}
A la suite de la découverte fortuite par un paysan, sur le territoire de Mackwiller, au licu-dit Hinler der Kirch (parcelles 40,41), d'un bloc en grès gris décoré d'imbrications, une fouille d'urgence fut exécutée non loin du site de la trouvaille. Une série de sondages fut d'abord pratiquée, qui permirent de fixer deux emplacements archéologiques intéressants : l'un à environ sept mètres à l'est du chemin de terre qui, partant du village et passant près des thermes gallo-romains et du cimetière, dessert les champs, l'autre à une cinquantaine de mètres à l'est du premier (fig. 1).

Sur le second emplacement, furent remarquées de nombreuses tuiles à rebords brisées et des traces de calcination dans le sol. Sur le premier, des débris de construction en pierre, deux blocs de grès paraissant en place et deux traits de calcination, bien marqués dans le sol, épais de $0,03 \mathrm{~m}$, délimitant un espace trapézoïdal de $3 \mathrm{~m}$ de longueur environ sur $1,60 \mathrm{~m}$ et $1,80 \mathrm{~m}$. Sur le premier emplacement, le plus proche du chemin de terre, devait être dégagé, en quinze jours, une fondation circulaire, d'un diamètre d'environ $7,50 \mathrm{~m}$, flanquée de huit contreforts et appuyée au sud sur une énorme fondation en blocs de grès massifs, longue de 4,75 m, large de $1 \mathrm{~m}$, profonde de $1,30 \mathrm{~m}$ (fig. 2). Curieusement, cette fondation avait été pourvue, à la profondeur de $0,70 \mathrm{~m}$, sous le sol actuel, d'une conduite cylindrique taillée en deux moitiés dans les deux séries de blocs de base, et qui ne correspondait à aucune ouverture pratiquée dans la maçonnerie du contrefort contre lequel elle s'appuie. Cette fondation a été construite en gros blocs taillés, à joints vifs, avec dispositif d'anathyrose. Les dimensions de ces blocs dépassent $2 \mathrm{~m}$ de longueur et $1 \mathrm{~m}$ de largeur. Leur épaisseur est comprise entre 0,35 et $0,65 \mathrm{~m}$. La destination de cette fondation massive pose un problème : a-t-elle servi à ancrer la fondation circulaire et à l'empêcher de glisser sur la pente ? Elle semble avoir été surmontée d'un autel monumental destiné au culte des morts.

Au milieu de la fondation circulaire, une fosse calcinée, dont les parois étaient visibles dès le début du sondage, immédiatement sous la couche arable, était orientée nord-sud. Elle présentait deux niveaux successifs de calcination : le premier situé à $0,30 \mathrm{~m}$ au-dessus du fond, le second constituant le fond même de la fosse (fig. 3). Ciette dernière, d'après les observations qui furent faites lors des fouilles, a été creusée et les deux feux ont brùlé, avant la construction de la fondation circulaire. En effet, au nord comme au sud de la fosse,
\end{abstract}




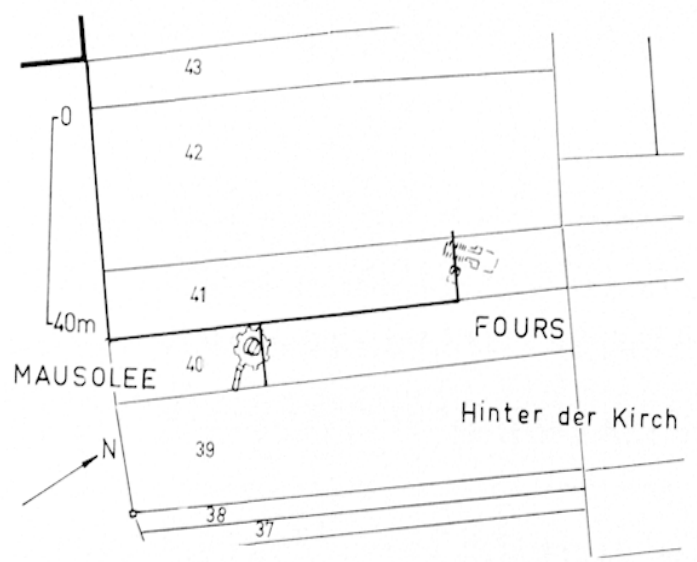

1 Plan de situation des fouilles de Mackwiller en 1966.

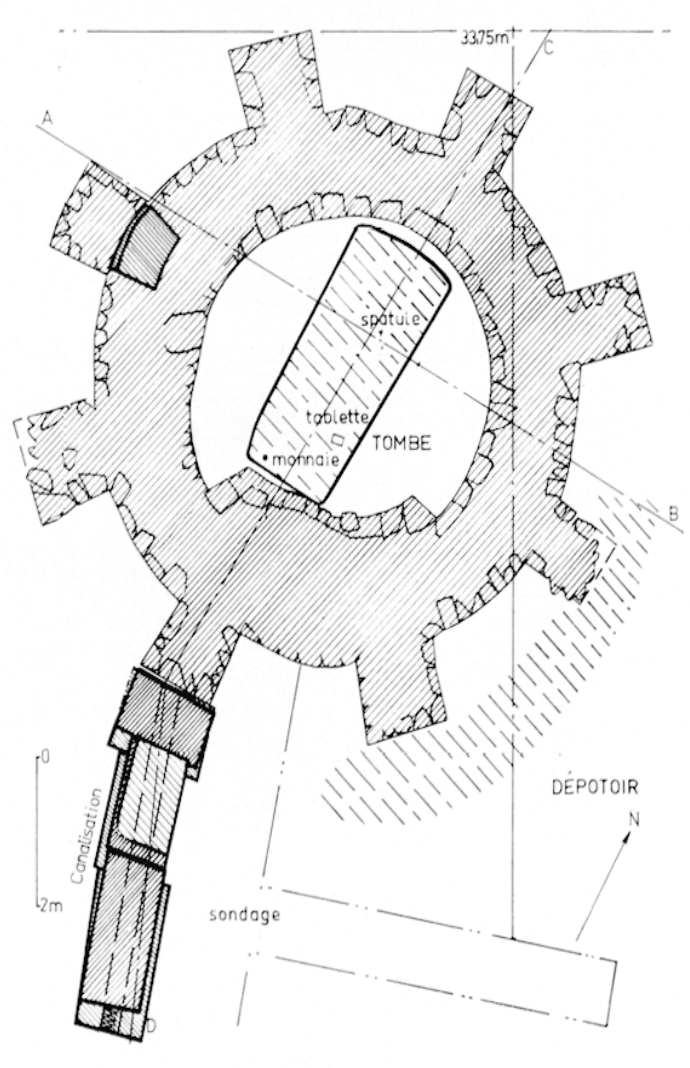

2 Plan du mausolée. $\rightarrow$
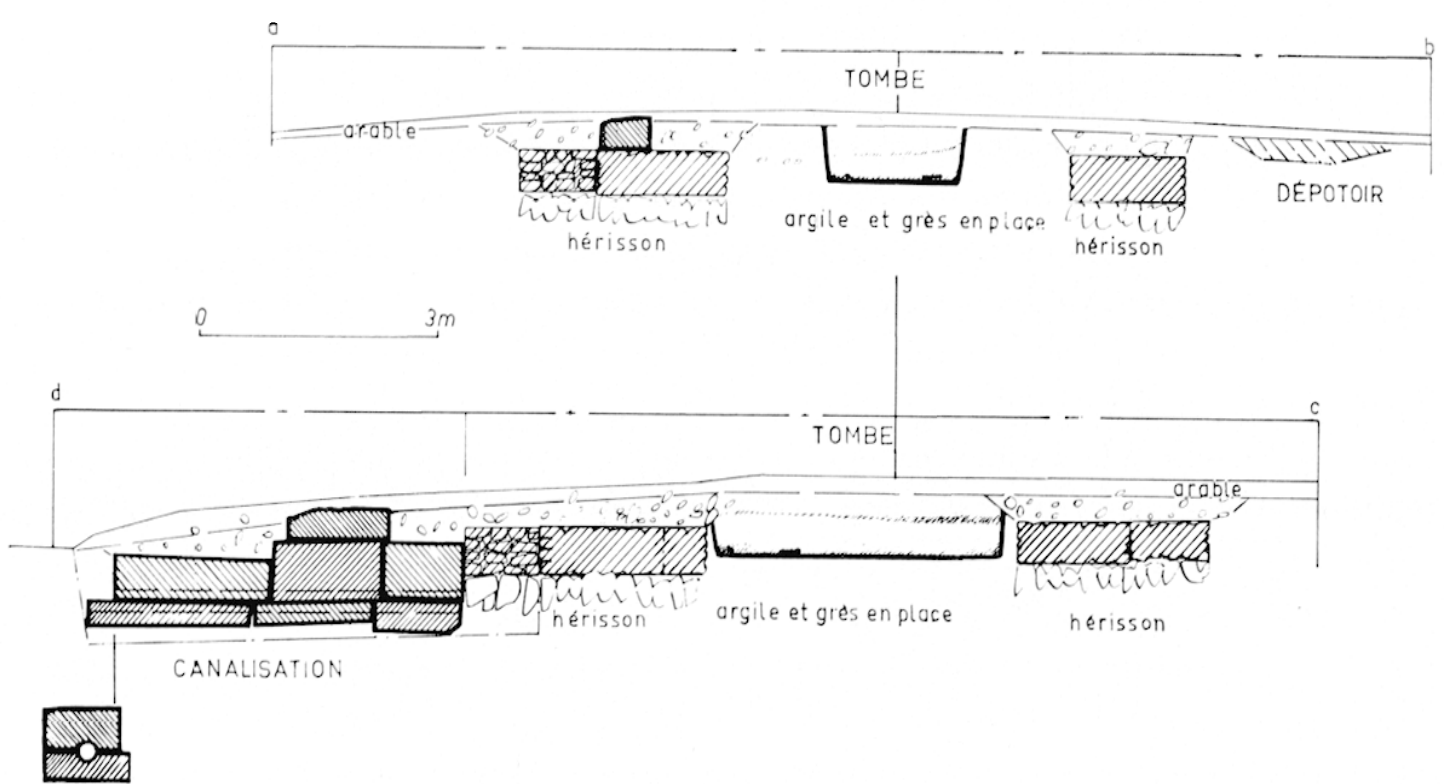

3 Coupe du mausolée.

on apercevait nettement que la terre calcinée ne touchait pas le mur mais qu'elle en était séparée par une mince couche de sable rose non calciné, ayant servi de liant aux blocs. Au sud, les blocs du mur situés à $0,02 \mathrm{~m}$ de la fosse calcinée ne présentaient aucune trace de chaleur.

Le monument circulaire possédait une fondation en moyen appareil en moellons de grès gris (fig. 4). Les moellons du parement, soigneusement appareillés, encadrent un 


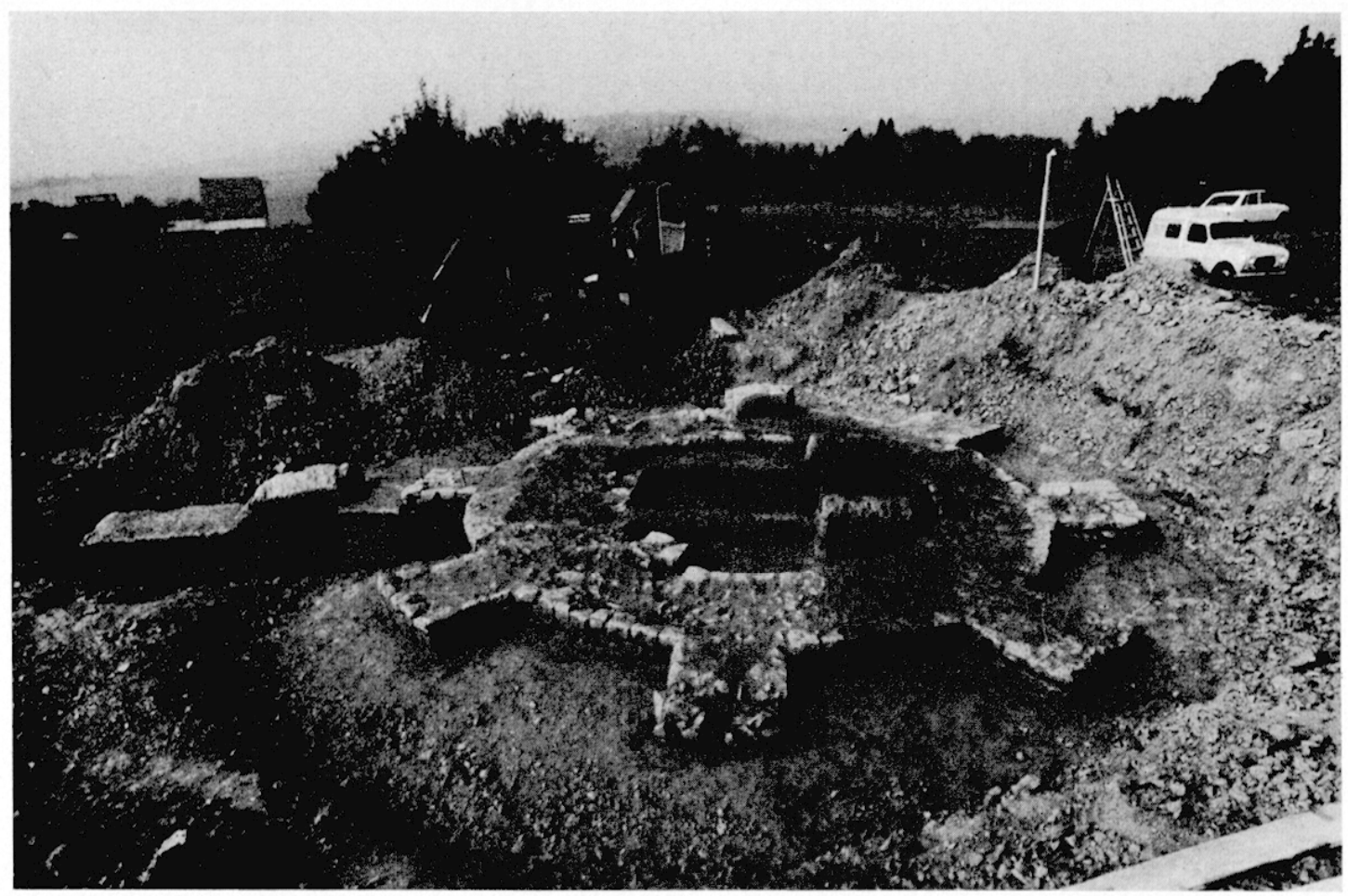

$a$

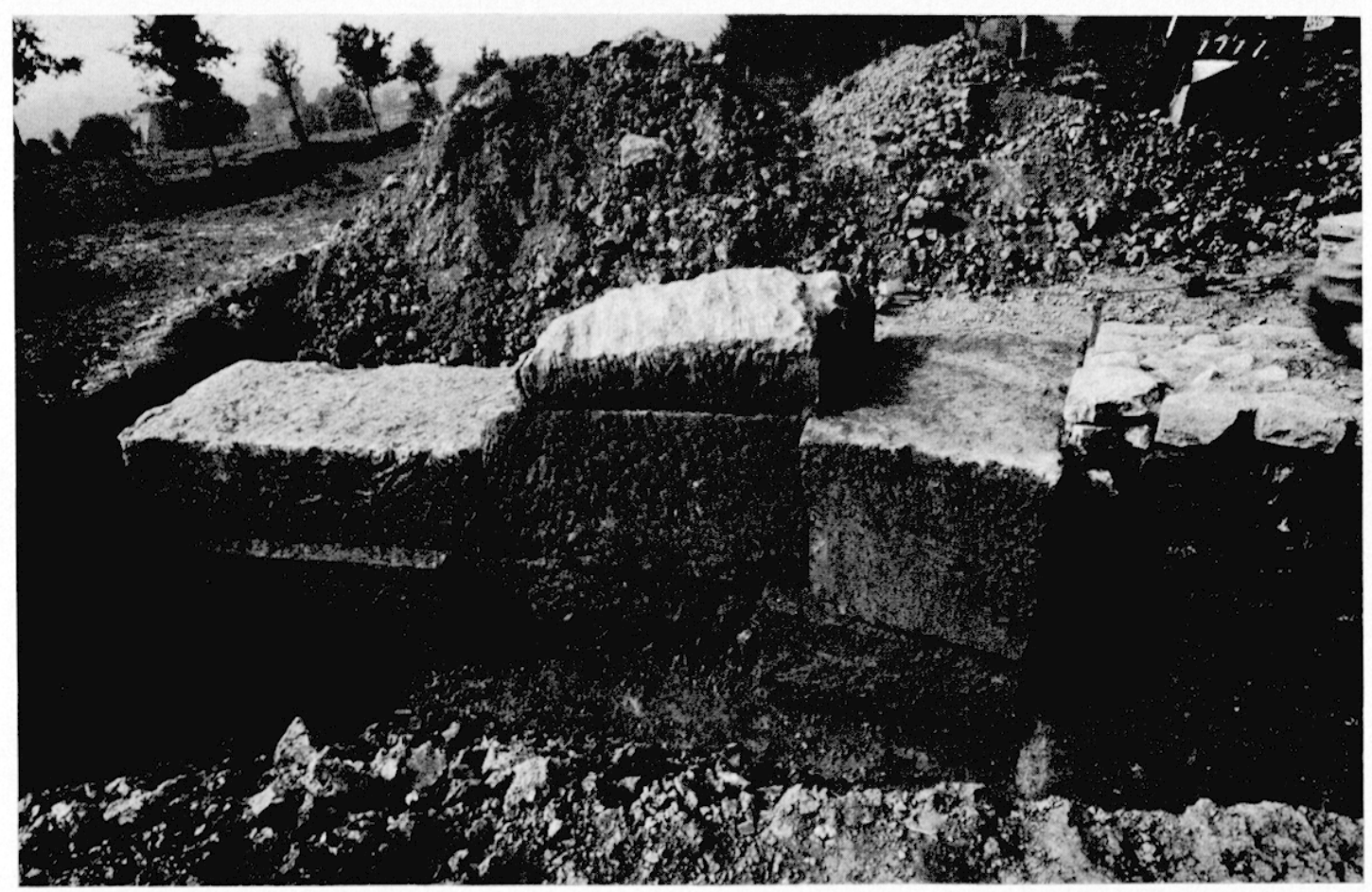

4 a) Vue générale des vestiges du mausolée. b) Détail : la canalisation. 
blocage intérieur de pierres et de sable rouge. A la base de la fondation, les blocs étaient placés verticalement. La profondeur actuelle de la fondation est, au nord, de $0,70 \mathrm{~m}$, au sud de 1,10 m. Elle s'épaississait fortement vers le sud, ce qui est normal, car le terrain est en pente. Au sud, un saillant interne marquait, dans la fondation, l'emplacement de la porte d'entrée. Le mur en œuvre, dont il reste un seul bloc encore en place, au niveau du contrefort ouest, était constitué de gros blocs de grès taillés à joints vifs. Ses faces intérieures présentaient le dispositif de l'anathyrose, déjà observé lors de la découverte du sanctuaire de Mithra. Le bloc conservé, taillé en claveau, présentait les dimensions suivantes : longueur à l'extérieur : $1 \mathrm{~m}$, à l'intérieur, $0,80 \mathrm{~m}$, les côtés mesurant $0,75 \mathrm{~m}$, la hauteur, incomplète, $0,35 \mathrm{~m}$.

Au-dessus du mur en gros blocs massifs, percé d'un conduit cylindrique à la base, était conservé un autre bloc d'appareil, dont les dimensions sont : longueur $1,10 \mathrm{~m}$, largeur $0,80 \mathrm{~m}$, hauteur actuelle, $0,35 \mathrm{~m}$. La hauteur primitive du bloc peut être reconstituée et évaluée à $0,70 \mathrm{~m}$, car des traces existent encore, sur l'une des faces, du trou de louve ayant servi à lever le bloc. Cé trou étant forcément au milieu (le bloc ayant été posé de champ), la dimension originelle du bloc était double. Au-dessus des fondations du mausolée, dans les remblais, ont été découverts des fragments de décor architectural : le morceau de toiture cônique, décoré d'imbrications ; - un débris de base de colonne de $0,35 \mathrm{~m}$ de diamètre ; un fragment, mal conservé, qui peut provenir de la volute d'un autel monumental (?); - un fragment de chapiteau de colonnes en forme de feuille d'acanthes, et un fragment de corniche décoré de la même façon; - un fragment de sculpture en haut-relief. Ces divers débris permettent de reconstituer le monument comme un mausolée en forme de tholos, couvert d'un toit cônique orné d'imbrications, décoré de huit colonnes ou demicolonnes d'ordre corinthien. A gauche en entrant, un autel monumental était décoré de sculptures.

\section{USTRINUM CENTRAL ET RESTES DE MOBILIER FUNÉRAIRE}

La fosse contenait, au fond, des ossements calcinés répandus sur toute la surface, les plus gros os se trouvant au nord, correspondant aux pieds du cadavre incinéré. Furent mis au jour également de nombreux morceaux de bronze à moitié fondu et calciné provenant peut-être de bijoux, des débris de verre fondu de couleur blanche ainsi qu'une tablette en schiste poli, les fragments d'une spatule brisée en bronze argenté, et une monnaie, grand bronze de Claude. A l'extéricur du monument, au sud-est, touchant les contreforts, fut découvert un dépotoir, formé par les vestiges des mobiliers funéraires et des urnes à incinération (fig. 5). Jadis placés dans la sépulture, ces restes avaient été projetés à l'extérieur, lors de la violation de ce dernier. Ces mobiliers comportaient : des débris de verre brisé, décoré, de couleur verte et marron, des clous, des ossements calcinés, des fragments de charbon de bois. A noter spécialement, une très abondante céramique commune, trois vases Drag. 37 de Salurninus et Salto de Chémery (Trajan-Hadrien, fig. 6), une vingtaine de vases de formes Drag. 32 et 40, en sigillée lisse dont les fonds portent une estampille anépigraphe de Chémery (fig. 7). Cette marque est en forme circulaire, décorée 


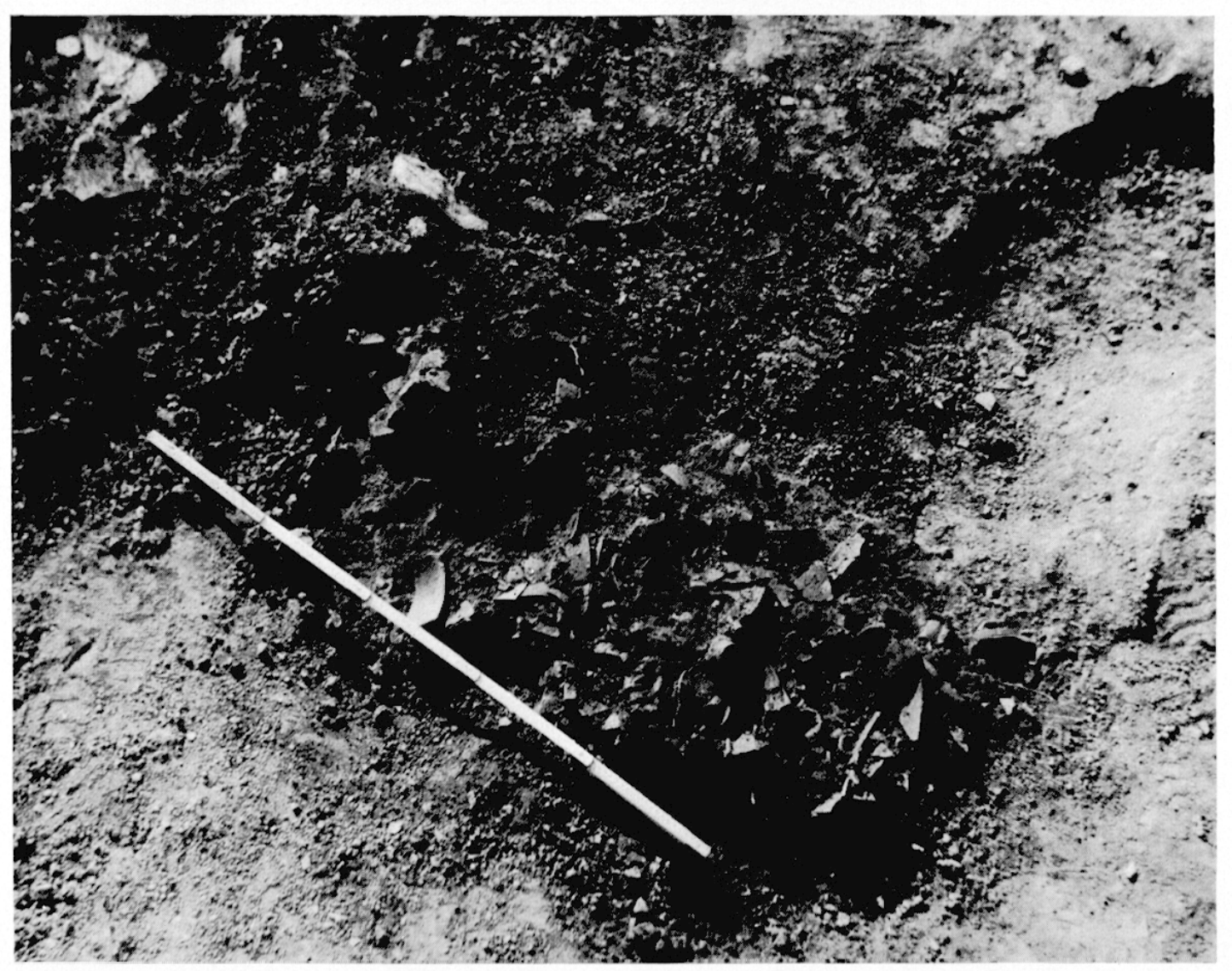

5 Le dépotoir : restes du mobilier funéraire.

extérieurement de neuf points et, à l'intérieur, d'un point central. Nous avons également observé la présence de plusieurs mortiers Drag. $38^{1}$.

A cinquante mètres à l'est, ont été dégagés deux fours (fig. 8 et 9). Le plus petit d'entre cux, orienté sud-nord, comportait seulement une chambre rectangulaire de $2 \mathrm{~m} \times$ $3 \mathrm{~m}$. Construites en tuiles plates assemblées avec de l'argile, ses parois étaient à peine calcinées. La sole de base était légèrement vitrifiée, cependant. Au nord, le four était fermé par un mur bien construit, en moyen appareil, qui ne portait pas de trace de calcination. A l'origine, le four était plus long, car les tuiles du fond se poursuivaient sous le second four. C'est donc après sa désaffectation qu'il a été limité par le mur et rempli de pierres en blocage, sans doute afin de servir de fondation à un autel du culte funéraire. C'est après la construction du grand four et en fonction de ce dernier, que ce nouveau dispositif a été édifié.

Le plus grand des fours comportait une chambre de combustion, de $3 \mathrm{~m} \times 3,75 \mathrm{~m}$, séparée en sept parties par des carneaux verticaux, bâtis en encorbellement avec des tuiles plates (fig. 8). Cés dernières avaient été fortement calcinées. La calcination s'arrête, vers l'extéricur, sur une ligne oblique, marquant le courant de chaleur. Un très long alandier, 

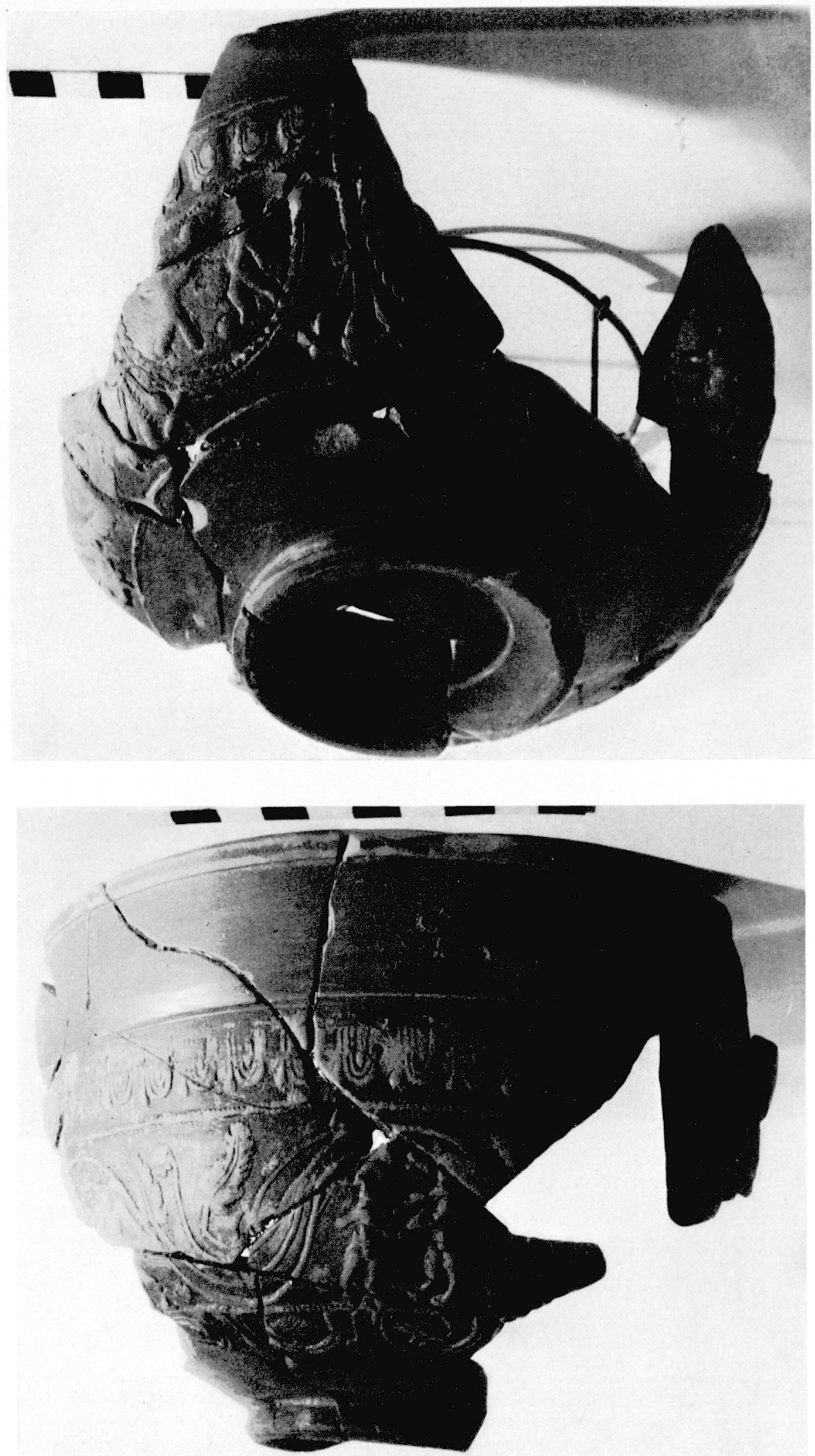

6 Dépotoir. Vases Drag. 37 reconstituables du maître Sallo-Salurninus. 

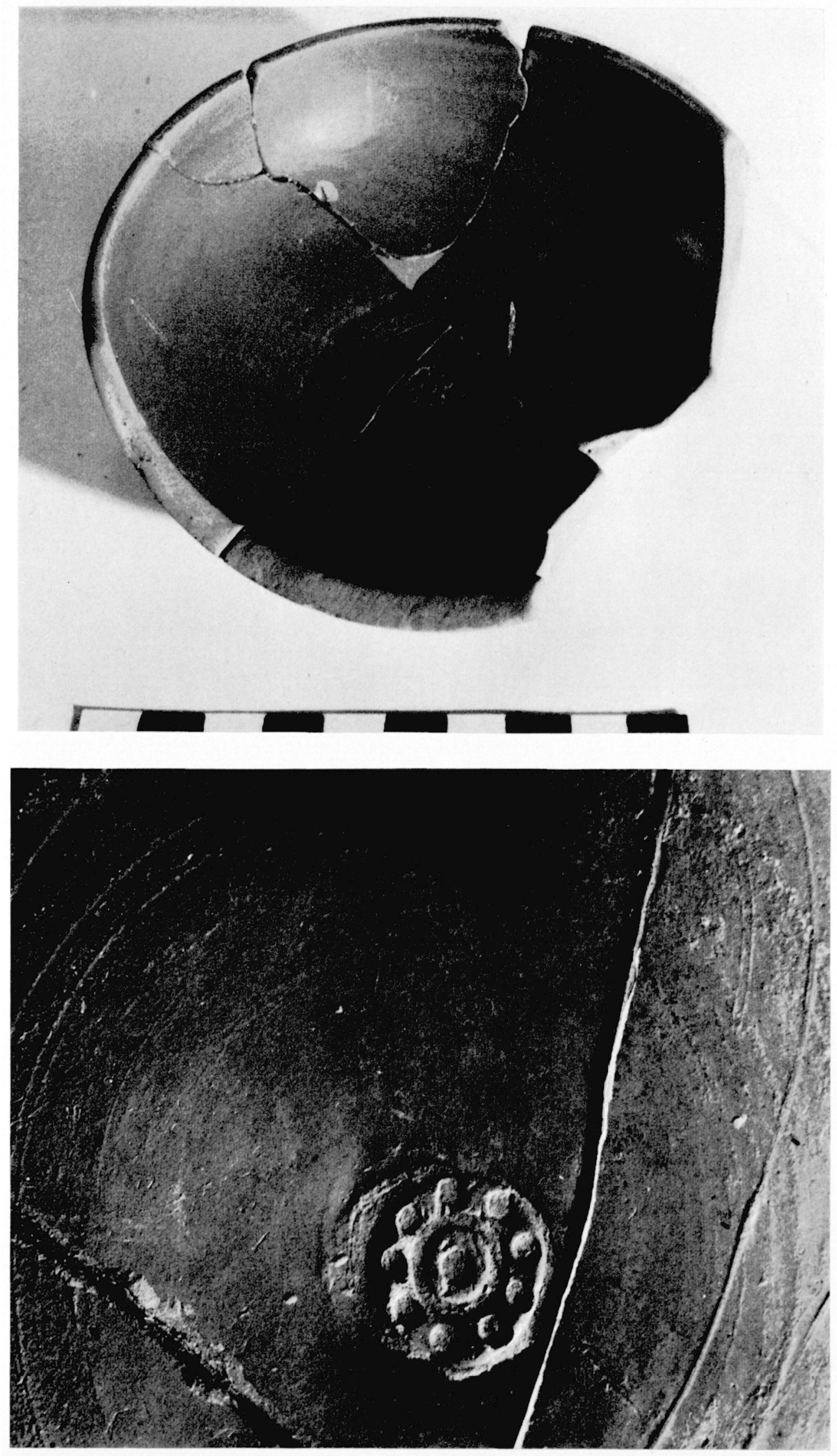

7 Dépotoir. Estampille d'un potier de Chémery sur le fond d'un petit vase Drag. 40. 
de 1,30 m sur 3,70 m, semble avoir été voûté à l'origine. A l'extrémité et de chaque côté de l'alandier, deux fondations en tuiles plates rectangulaires, larges de $1 \mathrm{~m}$., longues de $1,50 \mathrm{~m}$. Il y avait eu, entre ces sortes de contreforts en tuiles et les fondations de la chambre de combustion, un mur en blocs de grès non maçonnés, lutés avec de l'argile, dont subsistent seulement quelques restes, la majorité ayant été récupérée pour être utilisée, peut-être dès l'époque romaine.

Sur le fond de l'alandier, nous avons observé une couche épaisse de charbon de bois (sapin). A partir de l'entrée du four, cette couche s'arrêtait. Au beau milieu de la chambre de combustion du four, fut découverte une bûche calcinée, en bois plus dense (hêtre ou chêne). A l'entrée de l'alandier, a été dégagé un praefurnium fortement calciné, large de $3 \mathrm{~m}$, et long de $3 \mathrm{~m}$. Deux lignes de tuiles à rebord cernent à droite et à gauche cet emplacement, et attestent l'existence d'une toiture légère qui le couvrait. Le remblai du four, constitué exclusivement de tuiles, ne contenait pas de tessons. Seule fut découverte une pierre à aiguiser.

Le territoire de Mackwiller a déjà livré d'importants vestiges archéologiques d'époque romaine. En 1860, des fouilles exécutées par le Pasteur Ringel avaient mis au jour les fondations d'un balnéaire romain, attenant à une villa-palais. Lne partie de ces vestiges, les thermes, sont actuellement conservés et ouverts au public.

Entre 1953 et 1956, une découverte fortuite, dans une carrière de grès, située de l'autre côté de la rivière, mais toujours sur le territoire de la même commune, nous avait amené à exécuter des fouilles de sauvetage. Celles-ci devaient permettre de dégager les vestiges d'un sanctuaire de Mithra. A cette occasion, la déconverte d'une inscription nous a appris que le propriètaire du grand domaine et de la carrière de pierres romaine de Mackwiller était un chevalier romain. Un portrait assez réaliste découvert dans les ruines du sanctuaire de Vithra pourrait reproduire ses traits. Le Mithraeum de Mackwiller est daté par la céramique et les monnaies entre 150 et 160 ap. J.-C. Le style du haut relief de Mithra tauroctone qui y a été mis au jour est très apparenté à celui des sculptures de Koenigshoffen. Le mausolée découvert en 1966 à Mackwiller appartient à la première moitié du II ${ }^{\mathbf{e}}$ siècle. Il est donc plus ancien d'une génération que le sanctuaire de Mithra.

Le rite funéraire de la fosse à incinération, antérieure à la construction du mausolée lui-même, est fort remarquable et nous amène à penser à quelque survivance indigène. Observons que deux feux furent successivement allumés dans cette fosse : le premier pour incinérer le premier titulaire de la tombe, qui était probablement une femme, comme le prouvent les restes d'objets calcinés, le second, après l'incinération, pour purifier en quelque sorte l'emplacement.

Le mausolée lui-même, d'origine romaine, appartient à un type déjà connu à Neumagen au début du II $^{\mathrm{e}}$ siècle. La fondation massive, à gauche de l'entrée, reste énigmatique. Il est vraisemblable qu'elle supportait un autel, décoré de reliefs. La conduite qui y est pratiquée, à l'intérieur des blocs et en profondeur, ne laisse pas d'être assez surprenante. Son utilité pratique semble nulle. S'agit-il d'un dispositif purement symbolique, qui aurait été censé 


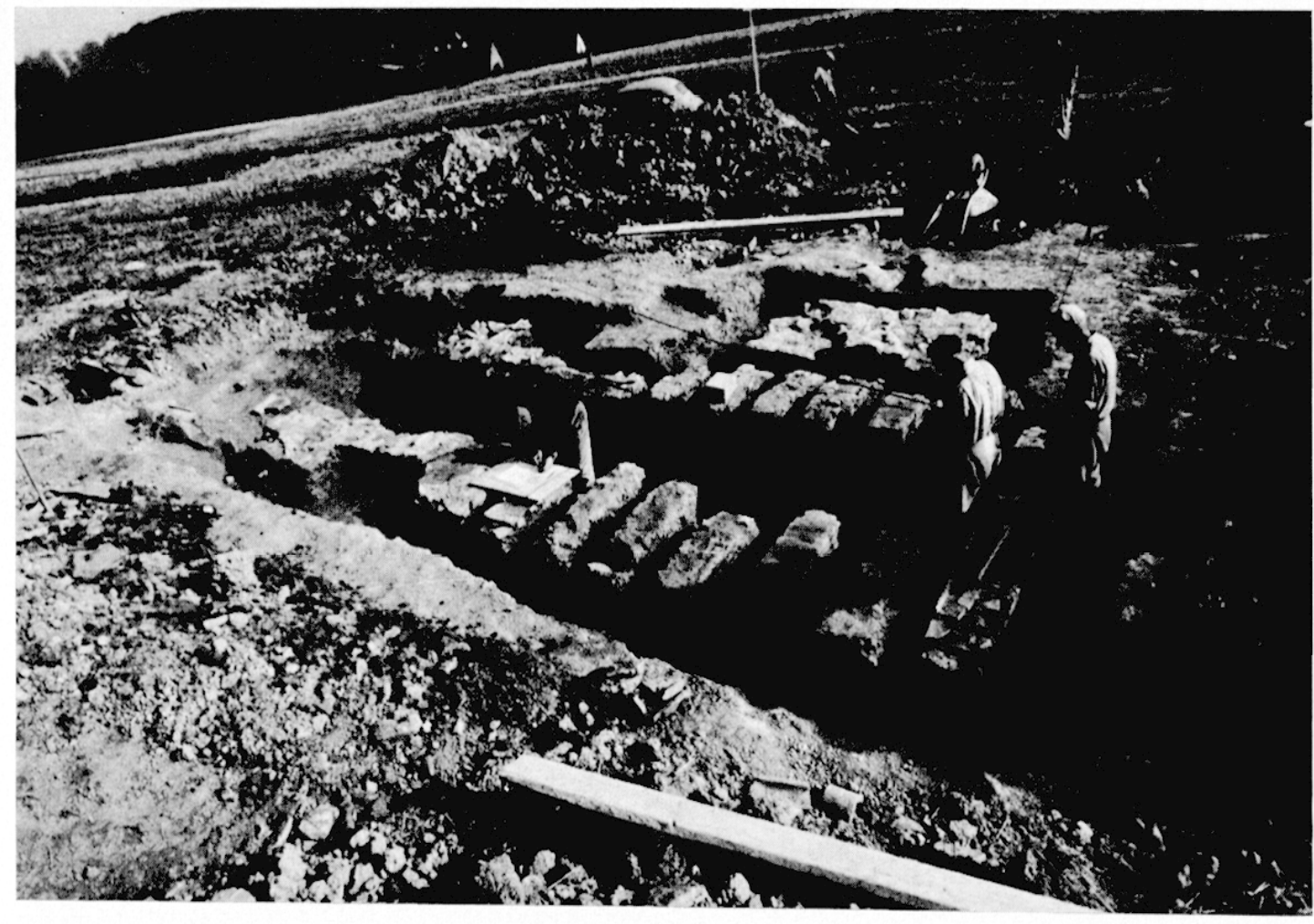

8 Vue générale du grand four.

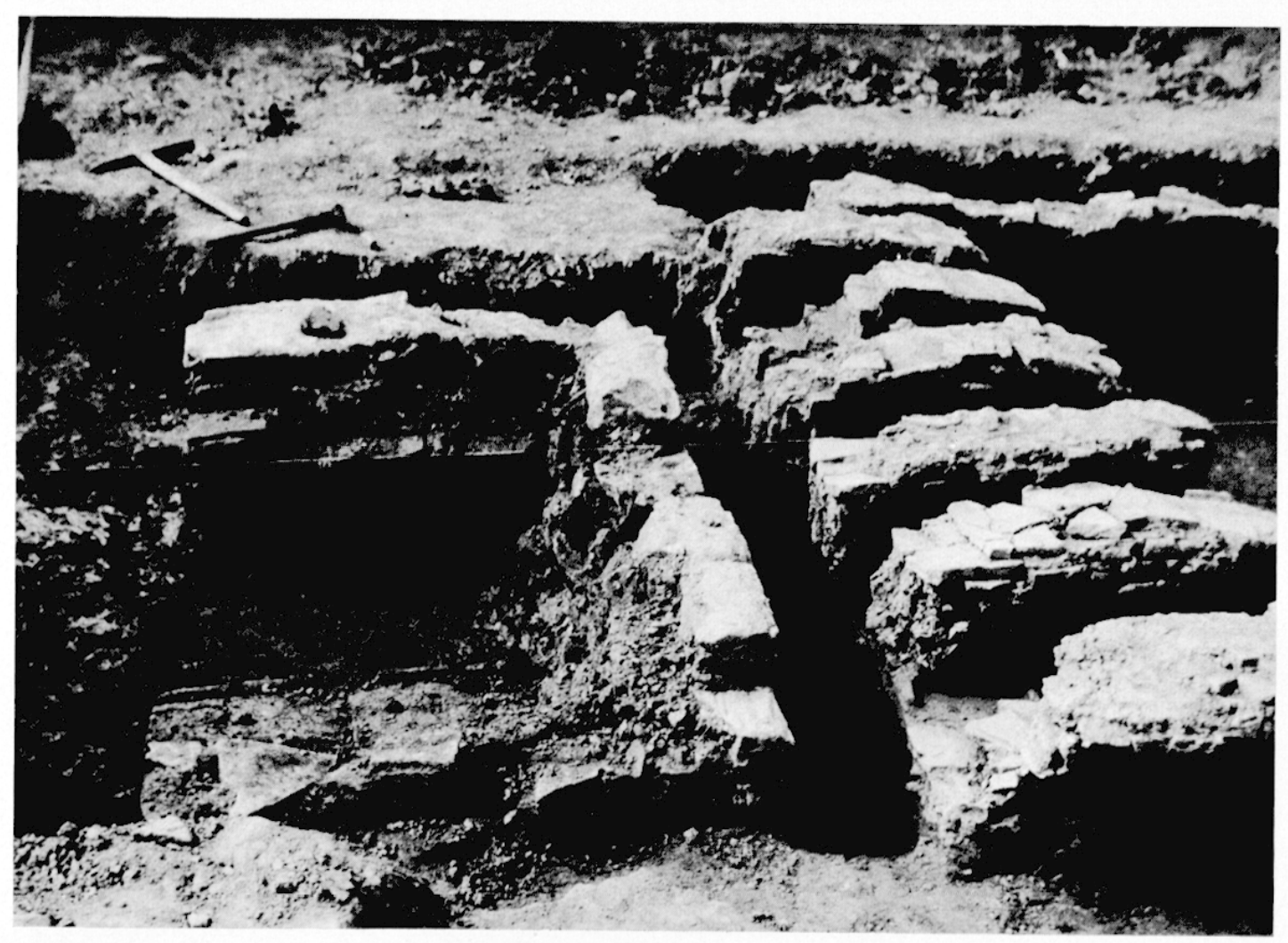

9 Les vestiges du petit four. 


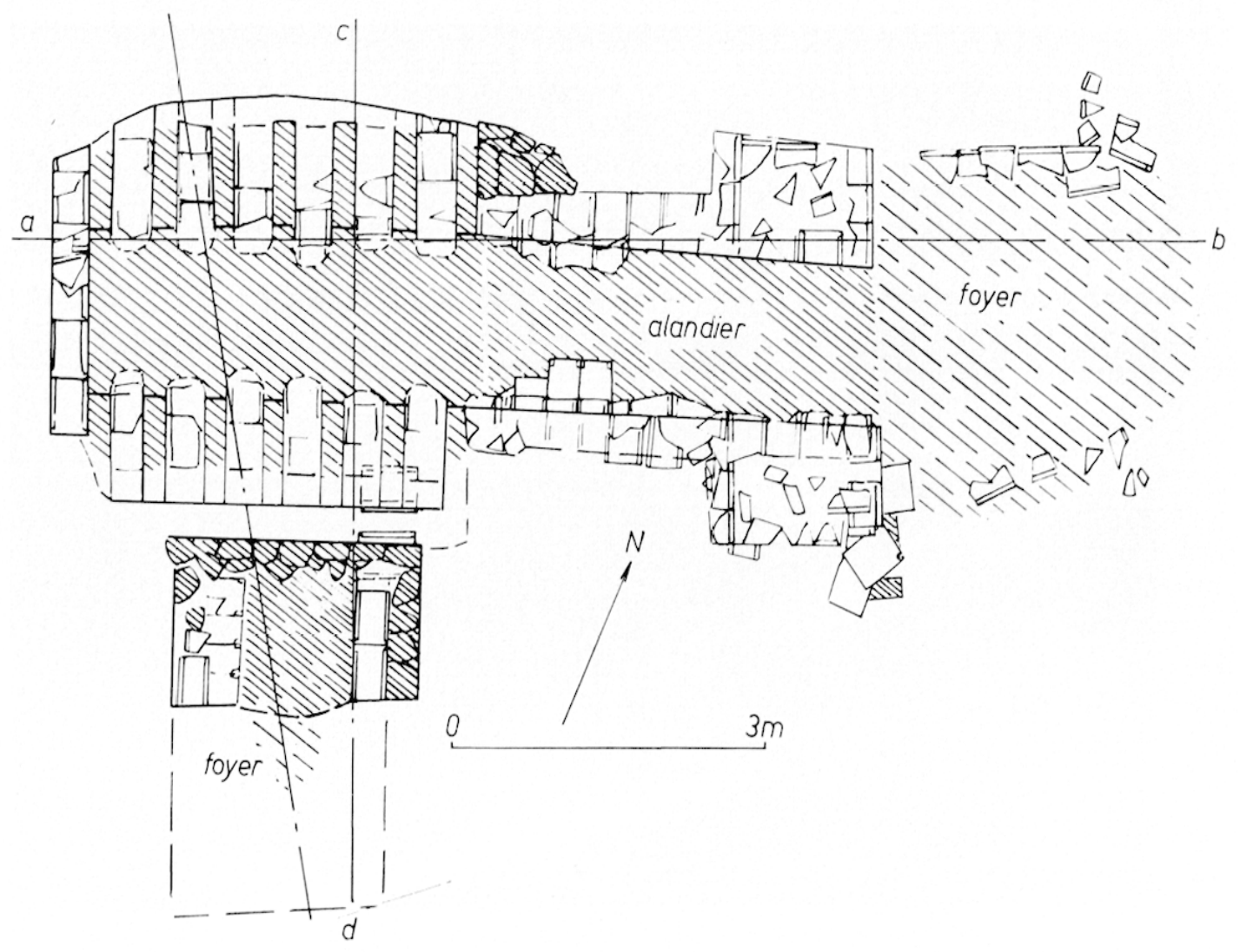

10 Plan de l'usirinum.

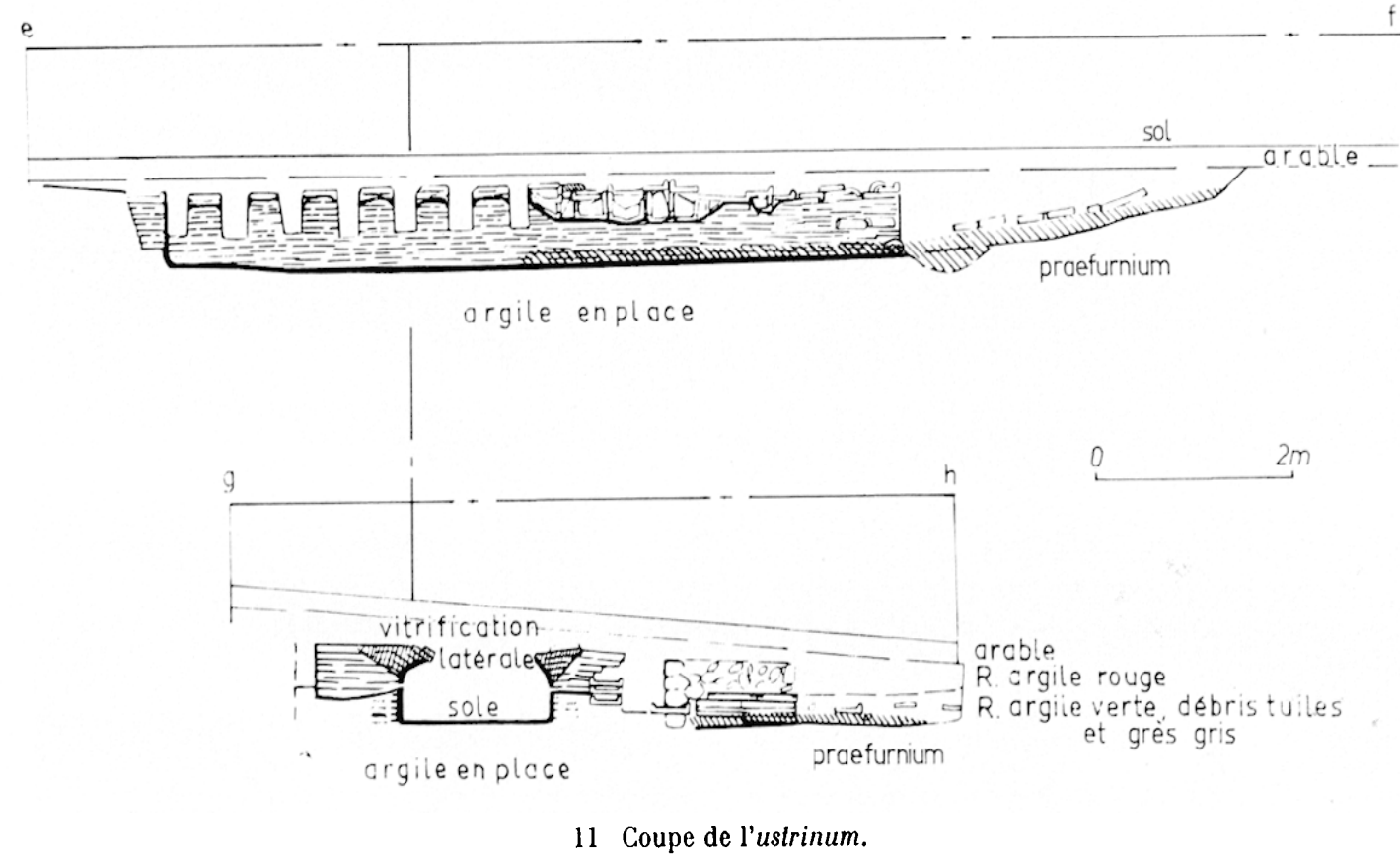


assurer la communication avec le monde souterrain, comme l'orifice pratiqué à la base des stèles maisons vosgiennes? Dans ce même domaine du rituel funéraire, nous remarquons que les fragmenls de vases sigillés, provenant des tombes anciennement placées dans le mausolée, sont en partie brûlés, en partie, sans trace de calcination. Il s'agit d'un usage funéraire assez curieux : le vase a été préalablement brisé, seulc la moitié des morceaux a passé par le bûcher, le reste ayant été réservé et placé après l'incinération dans la tombe. Quant aux fours mis au jour à cinquante mètres à l'est du mausolée, leur destination semble claire : il s'agit d'un uslrinum, d'une installation crématoire (fig. 10 et 11). L'installation primitive était constituée par un four de dimensions réduites et de construction sommaire, orienté comme la fosse à incinération du mausolée. Il semble avoir peu servi. Par la suite, il fut remplacé par un four plus grand, bâti sur le plan des fours de tuiliers du II $^{\mathrm{e}}$ siècle et orienté perpendiculairement. Et le premier four fut alors rempli de pierres et limité par un mur, pour servir de fondation à un autel destiné au culte.

Le second four permettait d'obtenir à l'intérieur de la chambre de chauffe une chaleur très considérable, la longueur de l'alandier voûté garantissant un tirage efficace. Cette nouvelle installation permettait de diminuer considérablement le temps exigé par l'incinération. Faut-il attacher une valeur symbolique au nombre de carneaux de ce four (sept), qui peut faire songer aux sept cyprès et aux sept autels enflammés figurant sur certains monuments mithriaques?

Les monuments funéraires du Haut-Empire nous sont surtout connus dans les enceintes urbaines où ils ont été employés, au Ive siècle, comme matériaux de construction. Il est rare de retrouver leurs fondations, plus rare encore, de mettre au jour des restes substantiels de leurs mobiliers funéraires permettant de les dater, encore plus exceptionnel, de pouvoir les mettre en rapport avec l'installation d'incinération qui les desservait. Pour ces trois raisons, les découvertes de Mackwiller présentent un caractère particulièrement intéressant.

Jean-Jacques HATT. 